\title{
2477. Adaptive machinery fault diagnosis based on improved shift-invariant sparse coding
}

\author{
Limin Li \\ Xi'an Polytechnic University, Xi'an, China \\ E-mail: liliminxiaomi@126.com
}

Received 18 August 2016; received in revised form 16 February 2017; accepted 12 March 2017

DOI https://doi.org/10.21595/jve.2017.17574

\begin{abstract}
In machinery fault diagnosis, it is common that one kind of fault may correspond to several conditions, these conditions may contain different loads, different speeds and so on. When using conventional intelligent machinery fault diagnosis methods on diagnosing this kind of faults, if only one condition among all of these conditions was trained, when using this trained classifier for diagnosing fault which containing all conditions, it would obtain a classification result with higher error, it is the problem of robustness; but if we train all these data in each condition, the robustness can be improved a lot, but the time would be wasted. In order to balance these two aspects of fault diagnosis which seem contradict with each other, someone proposed a new method which based on shift-invariant sparse coding (SISC) method, this method can learn features from each condition of the same fault, and these features are adaptive to other conditions, which solve the first problem, but time efficiency of this algorithm is lower, in this paper, by improving the efficiency of shift-invariant sparse coding, we can reduce a lot of time on learning features. Through the experiment testing, it showed that this new method proposed in this paper produced better performance than SISC algorithm.
\end{abstract}

Keywords: adaptive fault diagnosis, robustness and adaptive, shift-invariant sparse coding, improved shift-invariant sparse coding.

\section{Introduction}

At present, methods of machinery fault diagnosis can be divided into three categories, they are respectively modeling based methods, signal processing based methods and intelligent diagnosis methods [1-5]. Generally speaking, there are two main steps in fault diagnosis: the first step is feature extraction of vibration signals with some signal processing tools, and the second step is the fault classification based on the extracted features in the previous step $[6,7]$. How to extract features from vibration signals is one of the key problem in machine fault diagnosis. Since vibration signals usually contain non-stationary and nonlinear behaviors due to the complexity of the structure and work conditions, it is difficult to achieve an effective fault diagnosis only with the time domain or the frequency domain analysis. Hence features are extracted to indicate the characteristics of vibration signals. The conventional feature extraction methods include time-domain methods, frequency-domain methods, and time-frequency domain methods [8-10]. Features mentioned above are related to the fault, but not all of these features are useful for the classification result, so we have to select some features that are beneficial to the diagnosis result. These two works will not only waste a lot of time, while it would need several evaluation criterions to evaluate the classification result, so it is necessary to change this kind of mode for fault diagnosis.

Especially in the process of fault diagnosis, one problem that need pay attention is that for one machine, one kind of fault may correspond to several conditions, such as different loads, different speeds and so on, for example, inner race fault of a bearing can correspond to 4 different speeds, which would lead to two direct impacts, the first one is that if only one condition among all of these conditions was trained, it would get a classification result with high error while diagnosing other conditions of the same fault, it is the problem of robustness; but if we train all these data in each condition, the robustness can be improved a lot, but it sacrificed the time, because the quantity of the conditions is large. 
Recently, much attention has been paid on deep learning algorithm, because of its excellent feature learning ability, especially in the field of image processing [11] and speech recognition [12]. This kind of algorithm can overcome the disadvantage of traditional feature extraction methods by extracting the sparse representation of raw signal, and this sparse representation are learned from an over-complete dictionary, so it can represent the raw signal ideally. There are also someone who have applied some methods of deep learning on fault diagnosis, Alfredo Kalaitzis etc. proposed a novel convex scheme for simultaneous online fault classification and anomaly detection in a multivariate time-series setting[13], a sparse representation based approach for recognition of power system transients was proposed by Soumi Chakraborty etc. [14], Xuefeng Chen etc. proposed a compressed sensing based on dictionary learning for extracting impulse components, which learns a sparse dictionary from a whole noisy signal itself and then employs greedy algorithms to search impulse information in the learned sparse dictionary [15], a fusion sparse coding algorithm for impulse feature extraction in machinery weak fault detection was proposed by Sen deng etc., firstly, several sparse coding algorithms are executed in parallel independently as participating algorithms, then, fusion scheme of different sparse coding algorithms are presented to improve the accuracy of sparse signal reconstruction, lastly, the proposed method is used to process aircraft engine rotor vibration signals compared with other feature extraction approaches [16], Nishchal K. Verma etc. proposed a intelligent condition based monitoring method of rotating machines using sparse auto-encoders, sparse auto encoders have the ability to learn good features from the input data in an unsupervised fashion. Sparse auto-encoders and other deep architectures are already showing very good results in text classification, speaker and speech recognition and face recognition as well [17], Jipeng Xie etc. used deep belief network for learning features from high speed train vibration signals [18], Lingli Cui etc. proposed a matching pursuit of an adaptive impulse dictionary for bearing fault diagnosis, an adaptive matching pursuit algorithm that uses an impulse dictionary is introduced in this paper for rolling bearing vibration signal processing and fault diagnosis[19], Haifeng Tang etc. proposed a latent components analysis method based on sparse representation for machinery weak fault detection [20]. Algorithms above and some other paper [21, 22] which using learned sparse dictionary for machinery fault diagnosing, can diagnose different kind of fault with lower correct rate compared to EEMD algorithms, at the same time, it would take a lot of time when data from the same kind of fault which belong to different condition.

In order to solve problems mentioned above, an improve SISC framework for machinery fault diagnosis was proposed, this method can learn useful bases from just one condition of each kind of fault, but it can be adaptive to other conditions, so it can improve the robustness of fault diagnosis, meanwhile, we improved the shift-invariant sparse coding algorithm, which make it more efficient than before.

The remaining sections of this paper are listed as follows: Section 2 described the related work used in this paper, which introduced the algorithm of SISC; the proposed framework for adaptive fault diagnosis was stated in Section 3, which included improved shift-invariant sparse coding and novel framework for adaptive fault diagnosis; experimental test was given in Section 4; at last, we concluded this paper and made some discussion.

\section{Related work}

\subsection{Shift-invariant sparse coding}

SISC algorithm was firstly proposed by Monten Mørup etc., which is an extension of sparse coding which reconstructs one input data using all of the basis functions in all possible shifts [11].

In order to model SISC, additional parameters need to be introduced into the standard sparse coding model [23]: 
$X=\sum_{k} T_{l}\left(d_{k}\right) s_{k, l}+\varepsilon$,

where, $X$ is the input data; $d_{k}$ is the $k$ th dictionary; $s_{k, l}$ is the coefficient associated with the atom $d_{k}$ shifted for $l$ offsets; $\varepsilon$ is the observation noise with Gaussian distribution; $T_{l}$ is a shift operator which shifts an atom for $l \in[-L, L]$ offsets relatively.

Given a finite set of signals $X=\left\{X_{1}, X_{2}, \ldots, X_{N}\right\}$, assuming a uniform prior over the basis, a MAP estimator can be formulated for both bases and coefficients:

$\min _{d, s} \sum_{i=1}^{N}\left(\left\|X_{i}-\sum_{k=1}^{K} d_{k} * s_{k, i}\right\|_{2}^{2}+\beta \sum_{k=1}^{K}\left\|s_{k, i}\right\|\right)$,

Subject to $C \triangleq\left\{\left\|d_{k}\right\|_{2}^{2} \leq c, 1 \leq k \leq K\right\}$,

where $*$ represents convolution operator; $s_{k, i} \in R^{N-K+1}$ is the sparse activation of $d_{k}$ for signal $X_{i}, N$ is dimension of $X_{i}, K$ is number of dictionary, $\beta$ is penalty parameter of the sparsity constraint, $C$ is convex set of matrices which preventing $d_{k}$ from being very small. Specifically, $s_{k, i}$ can be solved in the convex optimization problem with $d_{k}$ being held fixed, and vice versa [24].

When using sparse coding algorithm on machinery fault diagnosing, it can diagnose some kinds of faults, but compared to traditional EEMD algorithm, it has lower correct rate, someone proposed using SISC algorithm to improve the correct rate of fault diagnosing, and gained some improvement [25], but meanwhile the computation complexity is added, so it is essential to reduce time which took by SISC algorithm, while not reducing the correct rate.

\section{Proposed framework for adaptive machinery fault diagnosis}

Adaptive machinery fault diagnosis algorithm is shown in Fig. 1. In order to reduce the diagnosis time and don't reduce robustness, we first need to learn dictionary for each kind of faults, then it is essential to combine all learned dictionaries into one over-complete dictionary, after shift invariant processing, by calculating the coefficients, we obtained all new features of each fault, when we need to diagnose a data set to see which fault it belongs, we need firstly to solve sparse representation, which is the new feature representation, using the trained classifier to diagnose the new data.

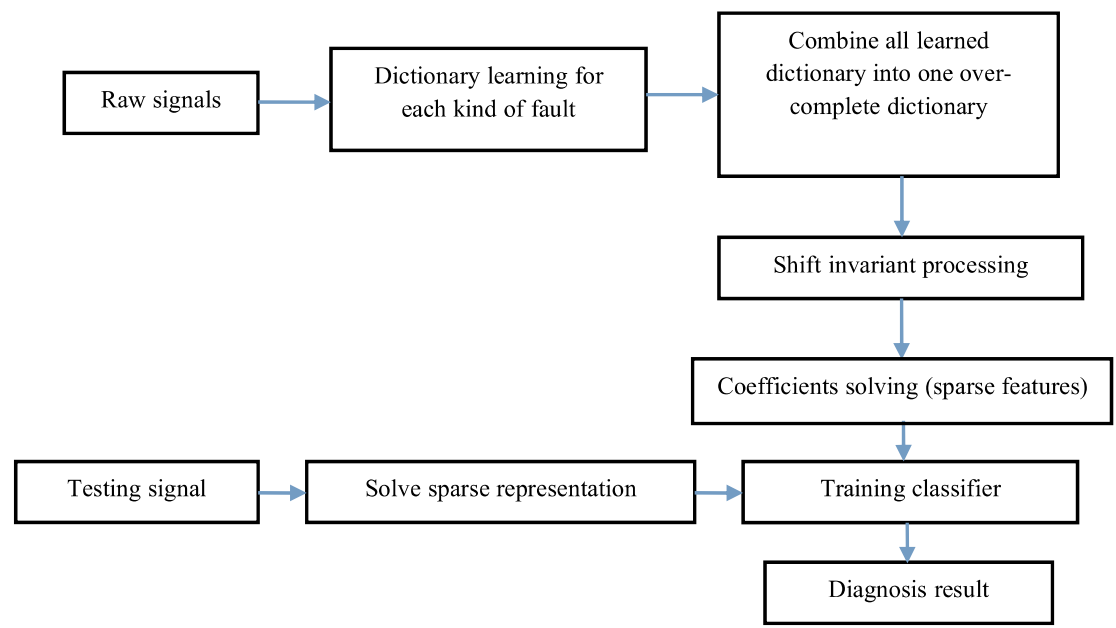

Fig. 1. Novel framework for machinery fault diagnosis 
In order to improve the computation performance of shift-invariant sparse coding algorithm, using the idea in literature [26] to solve the problem of SISC algorithm. Firstly, which holding $d_{k}$ fixed, solving $s_{k, i}$ by Eq. (3). Using feature-sign search algorithm for solving:

$\min _{s_{k, i}} \sum_{i=1}^{N}\left(\left\|X_{i}-\sum_{k=1}^{K} d_{k} * s_{k, i}\right\|_{2}^{2}+\beta \sum_{k}\left|s_{k, i}\right|\right)$.

Feature-sign search algorithm used for SISC:

(1) Initialize $s_{k}:=\overrightarrow{0}, \theta:=\overrightarrow{0}$, and "active set" : $=\{\}$, where $\theta_{i} \in\{-1,0,1\}$ denotes $\operatorname{sign}\left(s_{k, i}\right)$.

(2) From zero coefficients of $s_{k}$, select $i=\operatorname{argmax}_{i}\left|\frac{\partial\left\|X_{i}-d_{k} * s_{k}\right\|^{2}}{\partial s_{k, i}}\right|$, active $s_{k, i}$ (add $i$ to "active set") only if locally improves the objective, namely:

-If $\frac{\partial\left\|X_{i}-d_{k} s_{k}\right\|^{2}}{\partial s_{k, i}}>\beta$, then set $\theta_{i}:=-1$, "active set" : $=\{i\} \cup$ "active set".

- If $\frac{\partial\left\|X_{i}-d_{k} * s_{k}\right\|^{2}}{\partial s_{k, i}}<-\beta$, then set $\theta_{i}:=1$, "active set": $=\{i\} \cup$ "active set".

(3) Feature-sign step: Set $A=\sum_{k=1}^{K} d_{k}$, let $\hat{A}$ be a sub-matrix of $A$ that contains only the columns corresponding to the "active set".

Let $\hat{s}_{k, i}$ and $\hat{\theta}$ be sub-vector of $s_{k}$ and $\theta$ corresponding to the "active set".

Compute the analytical solution to the resulting unconstrained QP (minimize $\hat{s}_{k} \| X_{i}-$ $\left.d_{k} s_{k} \|^{2}+\beta \hat{\theta}^{T} \hat{s}_{k}\right)$ :

$\hat{S}_{k, n e w}:=\left(\hat{A}^{T} \hat{A}\right)^{-1}\left(\hat{A}^{T} X_{i}-\beta \hat{\theta} / 2\right)$,

Perform a discrete line search on the closed line segment from $\hat{s}_{k}$ to $\hat{s}_{k, \text { new }}$ :

Check the objective value at $\hat{s}_{k, n e w}$ and all points where any coefficient changes sign.

Update $\hat{s}_{k}$ (and the corresponding entries in $s_{k}$ ) to the point with the lowest objective value.

Remove zero coefficients of $\hat{s}_{k}$ from the active set and update $\theta:=\operatorname{sign}(x)$.

(4) Check the optimality conditions: (a) Optimality condition for nonzero coefficients: $\frac{\partial\left\|X_{i}-d_{k} s_{k}\right\|^{2}}{\partial s_{k, j}}+\beta \operatorname{sign}\left(s_{k, j}\right)=0, \forall s_{k, j} \neq 0$.

If condition (a) is not satisfied, go to step (3); else check condition (b).

(b) Optimality condition for zero coefficients: $\frac{\partial\left\|X_{i}-d_{k} s_{k}\right\|^{2}}{\partial s_{k, j}} \leq \beta, \forall s_{k, j}=0$.

If condition (b) is not satisfied, go to step (2); otherwise return $s_{k}$ as the solution.

Then holding $s_{k, i}$ fixed, solve $d_{k}$ by Eq. (4). Using the Lagrange dual for solving [23]:

$\min _{d_{k}}\left\|X_{i}-\sum_{k=1}^{K} d_{k} * s_{k, i}\right\|_{2}^{2}$

Subject to $\sum_{k=1}^{K} d_{k}{ }^{2} \leq c, \quad 1 \leq k \leq K$.

\section{Experimental testing}

\subsection{Experimental set-up}

Data used in this paper for experimental testing are provided by Case Western Reserve University (CWRU), the basic layout of the test rig is shown in Fig. 2. It consists of a $2 \mathrm{hp}$ reliance electric motor driving a shaft on which a torque transducer and encoder are mounted. Torque is applied to the shaft via a dynamo-meter and electronic control system. For these tests, faults 
ranging in diameter from 0.007 to 0.028 in. $(0.18-0.71 \mathrm{~mm})$ were seeded on the drive-end and fan-end bearings (SKF deep-groove ball bearings: 6205-2RSJEM and 6203-2RSJEM, respectively) of the motor using electro-discharge machining (EDM). The faults were seeded on the rolling elements and on the inner and outer races, and each faulty bearing was reinstalled (separately) on the test rig, which was then run at constant speed for motor loads of 0-3 horse power (approximate motor speeds of 1720-1797 rpm). Further details regarding the test set-up can be found at the CWRU Bearing Data Center website [27].

In order to test the improved SISC algorithm, 16 classes data were used, which include data in normal condition and in three fault condition, the used data are shown in Table 1 and Table 2.

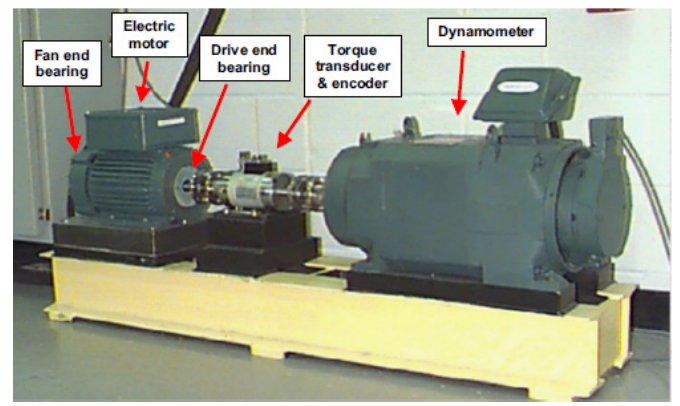

Fig. 2. CWRU bearing test rig

Table 1. Training data used in this paper (-: None)

\begin{tabular}{|c|c|c|c|c|c|c|c|}
\hline Fault diameter (in) & Load & Speed & Inner race & Ball & Outer_6 & Outer_3 & Outer_12 \\
\hline 0.007 & 0 & 1797 & IR007_0 & B007_0 & OR007_6_0 & OR007_3_0 & OR007_12_0 \\
\hline 0.014 & 0 & 1797 & IR014_0 & B014_0 & OR014_6_0 & - & - \\
\hline 0.021 & 0 & 1797 & IR021_0 & B021_0 & OR021_6_0 & OR021_3_0 & OR021_12_0 \\
\hline 0.028 & 0 & 1797 & IR028_0 & B028_0 & - & - & - \\
\hline 0 (N000, Normal) & 0 & 1797 & - & - & - & - & - \\
\hline
\end{tabular}

Table 2. Testing data used in this paper

\begin{tabular}{|c|c|c|c|c|c|c|c|}
\hline Fault diameter (in) & Load & Speed & Inner race & Ball & Outer_6 & Outer_3 & Outer_12 \\
\hline \multirow{5}{*}{0.007} & 1 & 1772 & IR007_1 & B007_1 & OR007_6_1 & OR007_3_1 & OR007_12_1 \\
\cline { 2 - 8 } & 2 & 1750 & IR007_2 & B007_2 & OR007_6_2 & OR007_3_2 & OR007_12_2 \\
\cline { 2 - 8 } & 3 & 1730 & IR007_3 & B007_3 & OR007_6_3 & OR007_3_3 & OR007_12_3 \\
\hline \multirow{5}{*}{0.014} & 1 & 1772 & IR014_1 & B014_1 & OR014_6_1 & OR014_3_1 & OR014_12_1 \\
\cline { 2 - 8 } & 2 & 1750 & IR014_2 & B014_2 & OR014_6_2 & OR014_3_2 & OR014_12_2 \\
\cline { 2 - 8 } & 3 & 1730 & IR014_3 & B014_3 & OR014_6_3 & OR014_3_3 & OR014_12_3 \\
\hline \multirow{5}{*}{021} & 1 & 1772 & IR021_1 & B021_1 & OR021_6_1 & OR021_3_1 & OR021_12_1 \\
\cline { 2 - 8 } & 2 & 1750 & IR021_2 & B021_2 & OR021_6_2 & OR021_3_2 & OR021_12_2 \\
\cline { 2 - 8 } & 3 & 1730 & IR021_3 & B021_3 & OR021_6_3 & OR021_3_3 & OR021_12_3 \\
\hline \multirow{5}{*}{0.028} & 1 & 1772 & IR028_1 & B028_1 & OR028_6_1 & OR028_3_1 & OR028_12_1 \\
\cline { 2 - 8 } & 2 & 1750 & IR028_2 & B028_2 & OR028_6_2 & OR028_3_2 & OR028_12_2 \\
\cline { 2 - 8 } & 3 & 1730 & IR028_3 & B028_3 & OR028_6_3 & OR028_3_3 & OR028_12__3 \\
\hline
\end{tabular}

\subsection{Test framework}

\subsubsection{Training results}

We use half data of load 0 for calculating dictionary representation and its corresponding coefficient, and use algorithm proposed in this paper for training, and at last corresponding dictionaries of each training data were calculated, which are shown in Fig. 3.

Dictionaries calculated by improved SISC algorithm are shown in Fig. 3, sparse features of 16 kinds of bearing faults were extracted, each dictionary composed of 8 bases, this series of 
dictionaries indicate different vibration signal can be represented by different dictionaries. Coefficients of each base composed of the sparse features, Table 3 is the classification error rate of training features extracted by SISC algorithm using SVM algorithm.

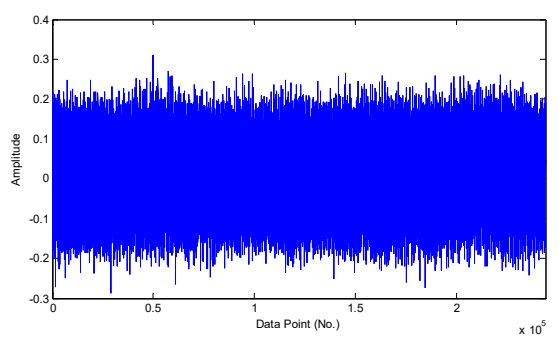

a) Data 1: (N000, Normal)
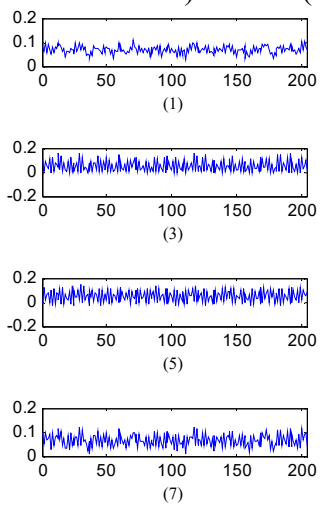

c) Data 1: Dictionary

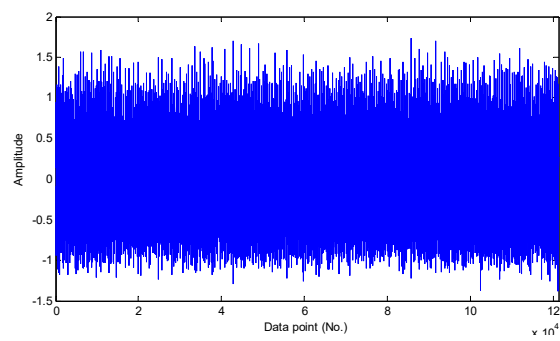

b) Data 2: (IR007_0)
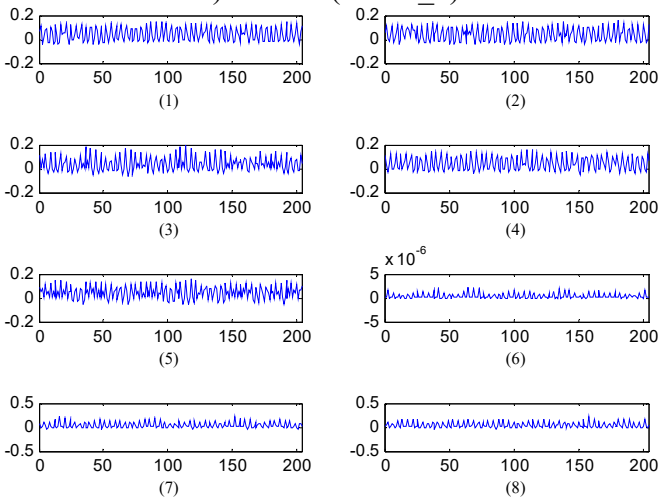

d) Data 2: Dictionary

Fig. 3. Raw signal of 2 of 16 kinds of bearing faults and their corresponding dictionary with one dictionary set of 204 shift variable dictionary and W:204×8×204, (length of dictionary* number of dictionaries * shift time) $x$-axis of dictionary: length of the base; $y$-axis of dictionary: amplitude of base

Table 3. Classification error rate of training data (-: None)

\begin{tabular}{|c|c|c|c|c|c|c|}
\hline \multirow{3}{*}{ Fault diameter (in) } & \multicolumn{6}{|c|}{ Classification error rates $(\%)$} \\
\hline & \multirow{2}{*}{ Normal } & \multirow{2}{*}{ Inner } & \multirow{2}{*}{ race ball } & \multicolumn{3}{|c|}{ Outer race position } \\
\hline & & & & Center@6:00 & Orthogonal@3:00 & Opposite @12:00 \\
\hline 0 & 0 & - & - & - & - & - \\
\hline 0.007 & - & 0.36 & 0.57 & 0 & 0 & 0.09 \\
\hline 0.014 & - & 0 & 1.86 & 1.2 & - & - \\
\hline 0.021 & - & 0.96 & 0.89 & 3.75 & 0 & 0.49 \\
\hline 0.028 & - & 1.24 & 1.76 & $\overline{-}$ & - & - \\
\hline
\end{tabular}

\subsubsection{Recognition correct rate between SISC and improved SISC algorithm}

After calculating dictionary of each kind of fault, we combine all those dictionaries into one over-complete dictionary, there are a variety of method for fusing child dictionaries into one over-complete dictionary according to the needs of signal sparse representation solution, the simplest way is by connecting directly parallel combination more child dictionaries [28]:

$D=\left[d_{1}, d_{2}, \ldots, d_{k}\right]$

where $d_{k}$ is child dictionary, $D$ is over-complete dictionary. Connect a parallel combination more child dictionaries to one to solve the coefficient of new kind of fault, it is easy to diagnosis the fault class by training classifier with sparse represent, which can be called sparse features, using all these sparse features to train classifier, and took other half data as testing data, in this paper, 
SVM classifier was used [29]. In order to compare recognition correct rate between SISC and improved SISC algorithm, using features extracted by SISC and improved SISC algorithm for testing, Table 4 are classification error rate of 60 groups test data.

From Table 4 we can conclude that when using improved SISC for classing machinery fault, the classification error rate is smaller than results which using SISC algorithm, so we can gain the conclusion that algorithm proposed in this paper improved the adaptive performance of SISC algorithm for fault diagnosing.

Table 4. Classification error rate of testing data between SISC and improved SISC (-: None; 1: Improved SISC, 2: SISC)

\begin{tabular}{|c|c|c|c|c|c|c|}
\hline \multirow{3}{*}{ Fault diameter (in) } & \multirow{3}{*}{ Load (Hp) } & \multicolumn{5}{|c|}{ Classification error rates (\%) } \\
\hline & & \multirow{2}{*}{ Inner race } & \multirow{2}{*}{ Ball } & \multicolumn{3}{|c|}{ Outer race position } \\
\hline & & & & Center@6:00 & Orthogonal@3:00 & Opposite $@ 12: 00$ \\
\hline \multirow{8}{*}{0.007} & 11 & 0 & 3.79 & 0.76 & 2.98 & 0 \\
\hline & $1 \_2$ & 30.00 & 50.22 & 25.78 & 34.87 & 55.13 \\
\hline & 21 & 0 & 1.78 & 0 & 1.67 & 0 \\
\hline & 22 & 60.54 & 44.76 & 55.34 & 23.20 & 34.76 \\
\hline & $3 \_1$ & 1.79 & 3.66 & 0 & 2.45 & 0 \\
\hline & 32 & 30.45 & 24.56 & 44.63 & 43.72 & 50.07 \\
\hline & 11 & 0.56 & 2.36 & 4.97 & - & - \\
\hline & 12 & 22.78 & 60.79 & 22.54 & - & - \\
\hline \multirow{6}{*}{0.014} & 21 & 20.65 & 2.98 & 0.65 & - & - \\
\hline & 22 & 67.65 & 33.52 & 32.16 & - & - \\
\hline & 31 & 40.20 & 1.88 & 2.76 & - & - \\
\hline & 32 & 60.98 & 23.43 & 44.72 & - & - \\
\hline & 11 & 1.34 & 23.26 & 5.47 & 0.78 & 5.63 \\
\hline & 12 & 23.15 & 45.23 & 50.12 & 64.23 & 54.16 \\
\hline \multirow{6}{*}{0.021} & 21 & 0.34 & 20.05 & 27.62 & 0 & 0 \\
\hline & 22 & 33.75 & 55.32 & 67.83 & 53.12 & 12.34 \\
\hline & $3 \_1$ & 3.78 & 20.08 & 25.09 & 1.54 & 1.32 \\
\hline & 32 & 45.20 & 67.39 & 30.65 & 44.91 & 27.63 \\
\hline & 11 & 1.76 & 4.02 & - & - & - \\
\hline & 12 & 43.78 & 23.89 & - & - & - \\
\hline \multirow{4}{*}{0.028} & 21 & 4.89 & 4.50 & - & - & - \\
\hline & 22 & 46.72 & 52.19 & - & - & - \\
\hline & $3 \_1$ & 1.87 & 10.34 & - & - & - \\
\hline & 32 & 22.19 & 70.52 & - & - & - \\
\hline
\end{tabular}

Table 5. The running time (in seconds) for shift-invariant sparse coding algorithm and improved shiftinvariant sparse coding algorithm for fault diagnosis (1: SISC, 2: improved SISC)

\begin{tabular}{|c|c|c|c|c|c|c|c|}
\hline \multirow{3}{*}{$\begin{array}{l}\text { Fault diameter } \\
\text { (in) }\end{array}$} & \multirow{3}{*}{$\begin{array}{l}\text { Load } \\
(\mathrm{Hp})\end{array}$} & \multirow{3}{*}{$\begin{array}{c}\text { Different } \\
\text { algorithms }\end{array}$} & \multicolumn{5}{|c|}{ Running time (s) } \\
\hline & & & \multirow{2}{*}{$\begin{array}{l}\text { Inner } \\
\text { race }\end{array}$} & \multirow[b]{2}{*}{ Ball } & \multicolumn{3}{|c|}{ Outer race position } \\
\hline & & & & & $\begin{array}{l}\text { Center } \\
\text { (a)6:00 }\end{array}$ & $\begin{array}{c}\text { Orthogonal } \\
\text { (a)3:00 }\end{array}$ & $\begin{array}{c}\text { Opposite } \\
\text { @ } 12: 00 \\
\end{array}$ \\
\hline \multirow{6}{*}{0.007} & 1 & 1 & 1.79 & 1.64 & 1.75 & 2.05 & 3.65 \\
\hline & 1 & 2 & 0.85 & 0.83 & 0.88 & 1.76 & 3.07 \\
\hline & 2 & 1 & 1.89 & 1.93 & 2.33 & 2.88 & 2.23 \\
\hline & 2 & 2 & 0.87 & 0.72 & 1.90 & 1.34 & 1.02 \\
\hline & 3 & 1 & 1.90 & 1.88 & 2.35 & 2.98 & 1.89 \\
\hline & 3 & 2 & 0.88 & 0.75 & 1.80 & 1.76 & 0.34 \\
\hline
\end{tabular}

\subsubsection{Computation complexity}

At the same time, the improved SISC algorithm has improved the efficiency of raw SISC algorithm, the running time (in seconds) for shift-invariant sparse coding algorithm and improved 
shift-invariant sparse coding algorithm has been listed in Table 5. In Table 5, we listed the running time of testing data 0.007 (fault diameter (in)), which include 15 group testing data.

From Table 5 we can conclude that the running time of improved shift-invariant sparse coding algorithm is less than the algorithm of shift-invariant sparse coding.

\section{Conclusions}

In this paper, in order to improve the adaptive performance and reduce the time used for training SISC, an improved algorithm was proposed, which using feature-sign search algorithm and Lagrange dual for solving the dictionaries and coefficients of SISC model, by experimental testing, we can obtain the result that improved SISC can meet the need of adaptive and computation complexity for fault diagnosis, which change the conventional fault diagnosis model, and obtain a better result. It improves the performance of machinery fault diagnosis in two aspect, the first one is that according to the machinery fault diagnosis in multi-condition, adaptive machinery fault diagnosis algorithm was proposed, and another one is that the computation performance SISC was improved. But there are still some problems we need to deeply consider, such as the diagnosis accuracy in this adaptive condition, although it is not a key problem at present.

\section{Acknowledgement}

The test data used in this paper is provided by Case Western Reserve University (CWRU). This research is supported by National Natural Science Foundation of China (No. 50975231), and also supported by Dr. Start-up Funding of Xi'an Polytechnic University: BS1506.

\section{References}

[1] Yaguo Lei, Jing Lin, Ming Zuo J., He Zhengjia Condition monitoring and fault diagnosis of planetary gearboxes: a review. Measurement, Vol. 48, 2014, p. 292-305.

[2] Bouamama Ould B., Biswas G., Loureiro R., Merzouki R. Graphical methods for diagnosis of dynamic systems: review. Annual Reviews in Control, Vol. 38, 2014, p. 199-219.

[3] Lei Yaguo, He Zhengjia, Zi Yanyang A new approach to intelligent fault diagnosis of rotating machinery. Expert Systems with Applications, Vol. 35, 2008, p. 1593-1600.

[4] Jiang Hongkai, Li Chengliang, Li Huaxing An improved EEMD with multiwavelet packet for rotating machinery multi-fault diagnosis. Mechanical Systems and Signal Processing, Vol. 36, 2013, p. 225-239.

[5] Gryllias K. C., Antoniadis I. A. A support vector machine approach based on physical model training for rolling element bearing fault detection in industrial environments. Engineering Applications of Artificial Intelligence, Vol. 25, 2012, p. 326-344.

[6] Li Wei, Zhu Zhencai, Jiang Fan, et al. Fault diagnosis of rotating machinery with a novel statistical feature extraction and evaluation method. Mechanical Systems and Signal Processing, Vols. 50-51, 2015, p. 414-426.

[7] Xin Xiong, Shixi Yang, Chunbiao Gan A new procedure for extracting fault feature of multi-frequency signal from rotating machinery. Mechanical Systems and Signal Processing, Vol. 32, 2012, p. 306-319.

[8] Jardine A. K. S., Lin D., Banjevic D. A review on machinery diagnostics and prognostics implementing condition-based maintenance. Mechanical Systems and Signal Processing, Vol. 20, Issue 7, 2006, p. 1483-1510.

[9] Sreejith B., Verma A. K., Srividya A. Fault diagnosis of rolling element bearing using time-domain features and neural networks. IEEE Region 10 Colloquium and the Third ICIIS, Kharagpur, India, 2008, p. 8-10.

[10] Feng Zhipeng, Liang Ming, Chu Fulei Recent advances in time-frequency analysis methods for machinery fault diagnosis: a review with application examples. Mechanical Systems and Signal Processing, Vol. 38, Issue 1, 2013, p. 165-205. 
[11] Mørup M., Schmidt M. N., Hansen L. K. Shift invariant sparse coding of image and music data. Journal of Machine Learning Research, 2008.

[12] Grosse Rogor, Raina Rajat, Kwong Helen, et al. Shift-invariant sparse coding for audio classification. Proceedings of the 23rd Conference on Uncertainty in Artificial Intelligence, 2007.

[13] Kalaitzis Alfredo, Nelson James D. B. Online Joing classification and anomaly detection via sparse coding. IEEE International Workshop on Machine Learning for Signal Processing, France, 2014.

[14] Soumi Chakraborty, Amitava Chatterjee, Swapan Kumar Goswami A sparse representation based approach for recognition of power system transients engineering. Applications of Artificial Intelligence, Vol. 30, 2014, p. 137-144.

[15] Chen Xuefeng, Du Zhaohui, Li Jimeng, et al. Compressed sensing based on dictionary learning for extracting impulse components. Signal Processing, Vol. 96, 2014, p. 94-109.

[16] Deng Sen, Jing Bo, Zhou Hongliang Fusion sparse coding algorithm for impulse feature extraction in machinery weak fault detection. Prognostics and System Health Management Conference, Hunan, 2014, p. 251-256.

[17] Verma Nishchal K., Gupta Vishal Kwnar, Sharma Mayank, et al. Intelligent condition based monitoring of rotating machines using sparse auto-encoders. IEEE Conference on Prognostics and Health Management (PHM), 2013, p. 1-7.

[18] Xie Jipeng, Yang Yan Learning features from high speed train vibration signals with deep belief networks. International Joint Conference on Neural Networks (IJCNN), China, 2014, p. 2205-2210.

[19] Cui Lingli, Wang Jing, Lee Seungchul Matching pursuit of an adaptive impulse dictionary for bearing fault diagnosis. Journal of Sound and Vibration, Vol. 333, 2014, p. 2840-2862.

[20] Tang Haifeng, Chen Jin, Dong Guangming Sparse representation based latent components analysis for machinery weak fault detection. Mechanical Systems and Signal Processing, Vol. 46, 2014, p. 373-388.

[21] Liu H., Liu C., Huang Y. Adaptive feature extraction using sparse coding for machinery fault diagnosis. Mechanical Systems and Signal Processing, Vol. 25, Issue 2, 2011, p. 558-574.

[22] Tang H., Chen J., Dong G. Sparse representation based latent components analysis for machinery weak fault detection. Mechanical Systems and Signal Processing, Vol. 46, Issue 2, 2014, p. 373-388.

[23] Blumensath T., Davies M. On shift-invariant sparse coding. Proceedings of 5th International Conference on Independent Component Analysis and Blind Signal Separation, Spain, 2004, p. 1205-1212.

[24] Liu H., Liu C., Huang Y. Adaptive feature extraction using sparse coding for machinery fault diagnosis. Mechanical Systems and Signal Processing, Vol. 25, Issue 2, 2011, p. 558-574.

[25] Zhu Huijie, Wang Xinqing, Rui Ting Multi scale shift invariant sparse coding for robust machinery diagnosing. Transactions of Beijing Institute of Technology, Vol. 1, 2016, p. 19-24.

[26] Lee H., Battle A., Raina R., et al. Efficient sparse coding algorithms. Advances in Neural Information Processing Systems, 2006, p. 801-808.

[27] Smith Wade A., Randall Robert B. Rolling element bearing diagnostics using the Case Western Reserve University data: a benchmark study. Mechanical Systems and Signal Processing, Vols. 64-65, 2015, p. 100-131.

[28] Liu Haining Sparse Coding Based Machine Conditon Recognition and Its Application in the Condition Monitoring of a Heavy Roller Grinder. Shanghai Jiaotong University, 2011.

[29] Zhang X., Liang Y., Zhou J., et al. A novel bearing fault diagnosis model integrated permutation entropy, ensemble empirical mode decomposition and optimized SVM. Measurement, Vol. 69, 2015, p. $164-179$.

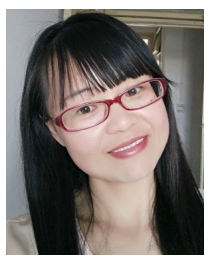

Limin Li received Ph.D. degree in School of Aeronautics from Northwestern Polytechnic University, Xi'an, China, in 2014. Now she works at Xi'an Polytechnic University. Her current research interests include fault diagnosis, embedded system development. 